\title{
The Collimated Jet Source in IRAS 16547-4247: Time Variation, Possible Precession, and Upper Limits to the Proper Motions along the Jet Axis
}

\section{Citation}

Rodríguez, Luis F., James M. Moran, Ramiro Franco-Hernández, Guido Garay, Kate J. Brooks, and Diego Mardones. 2008. "The Collimated Jet Source in IRAS 16547-4247: Time Variation, Possible Precession, and Upper Limits to the Proper Motions along the Jet Axis." Astronomical Journal 135 (6) (June 1): 2370-2379. doi:10.1088/0004-6256/135/6/2370.

\section{Published Version}

doi:10.1088/0004-6256/135/6/2370

\section{Permanent link}

http://nrs.harvard.edu/urn-3:HUL.InstRepos:11595849

\section{Terms of Use}

This article was downloaded from Harvard University's DASH repository, and is made available under the terms and conditions applicable to Other Posted Material, as set forth at http:// nrs.harvard.edu/urn-3:HUL.InstRepos:dash.current.terms-of-use\#LAA

\section{Share Your Story}

The Harvard community has made this article openly available.

Please share how this access benefits you. Submit a story.

Accessibility 


\title{
THE COLLIMATED JET SOURCE IN IRAS 16547-4247: TIME VARIATION, POSSIBLE PRECESSION, AND UPPER LIMITS TO THE PROPER MOTIONS ALONG THE JET AXIS
}

\author{
Luis F. Rodríguez ${ }^{1}$, James M. Moran ${ }^{2}$, Ramiro Franco-Hernández ${ }^{2,6}$, Guido Garay ${ }^{3}$, Kate J. Brooks ${ }^{4}$, and \\ Diego Mardones ${ }^{5}$ \\ ${ }^{1}$ Centro de Radioastronomía y Astrofísica, UNAM, Apdo. Postal 3-72, Morelia, Michoacán, 58089 Mexico, Mexico; 1.rodriguez@astrosmo.unam.mx \\ ${ }^{2}$ Harvard-Smithsonian Center for Astrophysics, 60 Garden Street, Cambridge, MA 02138, USA; jmoran@ @fa.harvard.edu, rfranco@cfa.harvard.edu \\ ${ }^{3}$ Departamento de Astronomía, Universidad de Chile, Casilla 36-D, Santiago, Chile; guido@ das.uchile.cl \\ ${ }^{4}$ Australia Telescope National Facility, P.O. Box 76, Epping NSW 1710, Australia; Kate.Brooks@atnf.csiro.au \\ ${ }^{5}$ Departamento de Astronomía, Universidad de Chile, Casilla 36-D, Santiago, Chile; mardones@ das.uchile.cl \\ Received 2007 October 21; accepted 2008 April 4; published 2008 May 14
}

\begin{abstract}
The triple radio source detected in association with the luminous infrared source IRAS 16547-4247 has previously been studied with high angular resolution and high sensitivity with the Very Large Array at $3.6 \mathrm{~cm}$ wavelength. In this paper, we present new $3.6 \mathrm{~cm}$ observations taken 2.68 years after the first epoch that allow a search for variability and proper motions, as well as the detection of additional faint sources in the region. We do not detect proper motions along the axis of the outflow in the outer lobes of this source at a $4 \sigma$ upper limit of $\sim 160 \mathrm{~km} \mathrm{~s}^{-1}$. This suggests that these lobes are probably working surfaces where the jet is interacting with a denser medium. However, the brightest components of the lobes show evidence of precession, at a rate of $0.08 \mathrm{yr}^{-1}$ clockwise in the plane of the sky. It may be possible to understand the distribution of almost all the identified sources as the result of ejecta from a precessing jet. The core of the thermal jet shows significant variations in flux density and morphology. We compare this source with other jets in low- and high-mass young stars and suggest that the latter can be understood as a scaled-up version of the former.
\end{abstract}

Key words: astrometry - ISM: individual (IRAS 16547-4247) - radio continuum: ISM - stars: formation

\section{INTRODUCTION}

A successful model of low-mass star formation, based on accretion via a circumstellar disk and a collimated outflow in the form of jets (Shu et al. 1987), has been developed and found to be consistent with the observations. An important question related to star formation is whether or not this model can be scaled up for the case of high-mass protostars or if other physical processes (i.e., stellar merging; Bonnell et al. 1998; Stahler et al. 2000; Bally \& Zinnecker 2005) are important. A small number of B-type young stars have been found to be associated with collimated outflows and possibly even circumstellar disks (see Garay \& Lizano 1999; Arce et al. 2007; Cesaroni et al. 2007). The source IRAS $16547-4247$ is the best example of a highlycollimated outflow associated with an O-type protostar studied so far, and its study may reveal important information about the way high-mass stars form.

The systemic LSR velocity of the ambient molecular cloud where IRAS $16547-4247$ is embedded is $-30.6 \mathrm{~km} \mathrm{~s}^{-1}$ (Garay et al. 2007). Adopting the galactic rotation model of Brand \& Blitz (1993) and assuming that the one-dimensional root-mean square (rms) velocity dispersion among molecular clouds is $7.8 \mathrm{~km} \mathrm{~s}^{-1}$ (Stark \& Brand 1989), we estimate a distance of $2.9 \pm 0.6 \mathrm{kpc}$ for the source. IRAS $16547-4247$ has a bolometric luminosity of $6.2 \times 10^{4} L_{\odot}$, equivalent to that of a single O8 zero-age main-sequence star, although it is probably a cluster for which the most massive star would have a slightly lower luminosity. Garay et al. (2003) detected an embedded triple radio continuum source associated with the IRAS 16547-4247. The three radio components are aligned in a northwest-southeast direction, with the outer lobes symmetrically separated from the central source by an angular distance of $\sim 10^{\prime \prime}$, equivalent to a

\footnotetext{
${ }^{6}$ Centro de Radioastronomía y Astrofísica, UNAM, Morelia 58089, México.
}

physical separation in the plane of the sky of $\sim 0.14 \mathrm{pc}$. The positive spectral index of the central source is consistent with that expected for a radio thermal (free-free) jet (e.g., Anglada 1996; Rodríguez 1997), while the spectral index of the lobes suggests a mix of thermal and nonthermal emission. Forster \& Caswell (1989) detected both mainline $\mathrm{OH}$ and $\mathrm{H}_{2} \mathrm{O}$ masers at a position close to the central continuum source. The triple system is centered on the position of the IRAS source and is also coincident within the measurement error with a $1.2 \mathrm{~mm}$ dust continuum and a molecular line emission core whose mass is on the order of $10^{3} M_{\odot}$ (Garay et al. 2003). Brooks et al. (2003) reported a chain of $\mathrm{H}_{2} 2.12 \mu \mathrm{m}$ emission knots that trace a collimated flow extending over $1.5 \mathrm{pc}$ that emanates from close to the central component of the triple radio source and has a position angle (P.A.) very similar to that defined by the outer lobes of the triple radio source. Most likely this extended component traces gas ejected in the past by the central component of the triple source. The molecular observations of Garay et al. (2007) revealed the presence of a collimated bipolar outflow with lobes $\sim 0.7 \mathrm{pc}$ in extent and aligned with the thermal jet located at the center of the core.

In a high-angular-resolution study made with the Very Large Array (VLA) and Australia Telescope Compact Array (ATCA), Rodríguez et al. (2005) confirmed that the central object is a thermal radio jet, while the two outer lobes are most probably heavily obscured $\mathrm{HH}$ objects. The thermal radio jet was resolved angularly for the first time by these authors and found to align closely with the outer lobes. Several fainter sources detected in the region away from the main outflow axis were interpreted as most probably associated with other stars in a young cluster. Brooks et al. (2007) used ATCA observations to substantiate the jet nature of the central source and to detect emission at $88 \mathrm{GHz}$ that most probably arises from dust associated with this source. 
In this paper, we present new sensitive high-angularresolution VLA observations that provide new information on the characteristics of the radio triple source in IRAS 16547-4247 as well as other sources in the field.

\section{OBSERVATIONS}

The new $3.6 \mathrm{~cm}$ wavelength observations were made using the VLA of the National Radio Astronomy Observatory (NRAO). ${ }^{7}$ These VLA radio continuum observations were carried out in 2006 May 31 and June 8 at the frequency of $8.46 \mathrm{GHz}$. The array was in the BnA configuration and an effective bandwidth of $100 \mathrm{MHz}$ was used. The absolute amplitude calibrator was $1331+305$ (with an adopted flux density of $5.21 \mathrm{Jy}$ ), and a source model provided by NRAO was used for its calibration. The phase calibrator was 1626-298, with bootstrapped flux densities of $1.684 \pm 0.003$ and $1.823 \pm 0.004 \mathrm{Jy}$ for the first and second epochs, respectively. The phase center of the array was $\alpha=16^{\mathrm{h}} 58^{\mathrm{m}} 17^{\mathrm{s}} .202$ and $\delta=-42^{\circ} 52^{\prime} 09^{\prime \prime} .59$ (J2000.0). The data were edited and calibrated using the software package Astronomical Image Processing System (AIPS) of NRAO. To correct for amplitude and phase errors caused by the low elevation of the source, the data were self-calibrated in phase and amplitude. No significant variations were found between the two epochs of observations (separated by only eight days) and the final analysis was made from the result of concatenating all data. The average epoch for the two data sets is 2006.42, which we use in subsequent analysis. Clean maps were obtained using the task IMAGR of AIPS with the ROBUST parameter set to 0. The synthesized (FWHM) beam was $1^{\prime \prime} .18 \times 00^{\prime} .60$; P.A. $=8^{\circ}$. The noise level achieved in the final image was $23 \mu \mathrm{Jy}_{\text {beam }}{ }^{-1}$.

The $3.6 \mathrm{~cm}$ wavelength archive data were taken on 2003 September 25 and 30 (epoch 2003.74) in very similar conditions to those of the new observations and the resulting beam was $1^{\prime \prime} .17 \times 0$ 0.64; P.A. $=11^{\circ}$. This old data set is described in Rodríguez et al. (2005). These data were cross calibrated in phase and amplitude using the 2006.42 epoch as the model, which serves to globally minimize the differences in positions and flux densities of the sources. The absolute flux-density error is estimated to be $10 \%$.

\section{RESULTS AND DISCUSSION}

To allow a more reliable comparison between the two epochs, both images were convolved to the same angular resolution $\left(1^{\prime \prime} .20 \times 0^{\prime \prime} .65\right.$; P.A. $\left.=9^{\circ}\right)$. To improve the relative gain calibration between the two epochs we examined the relative strengths of the 13 sources we identified in the images. It is clear that the ratios of the flux densities of all the sources except $\mathrm{S}-1$ and $\mathrm{N}-1$ had the same constant value of $0.91 \pm 0.02$. Hence, we adjusted the flux scale of the first epoch by this relative gain factor. Figure 1 shows the corrected ratio of the flux densities of all the sources. The reduced $\chi^{2}$ of the estimate of the gain constant is 0.76 , which suggests that the flux-density errors may be slightly overestimated by about 15 percent. Since the $\chi^{2}$ is close to unity we conclude that there is no evidence for variability among the 11 sources, whereas the changes in flux densities of S-1 and the jet are highly significant. Figure 2 shows contour images of the emission observed at $8.46 \mathrm{GHz}$ in the two epochs, as well as the difference image (2006.42-2003.74). The positions, flux densities, and deconvolved angular sizes of the sources

\footnotetext{
7 NRAO is a facility of the National Science Foundation operated under cooperative agreement by Associated Universities, Inc.
}

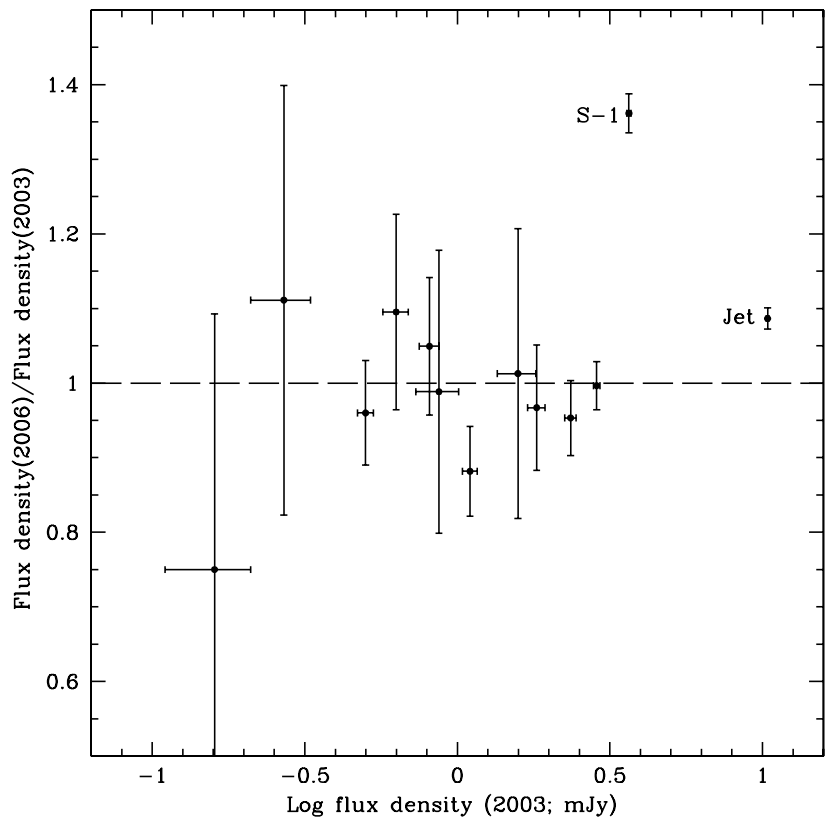

Figure 1. Ratio of 2006 to 2003 flux densities versus the logarithm of the 2003 flux density, given in mJy. Only the jet and source S-1 show clear evidence of variability between the two epochs.

identified in Figure 2 are given in Table 1. The observed parameters for each component were determined from a linearized least-squares fit to a Gaussian ellipsoid function using the task JMFIT of AIPS. In Table 2 we summarize the parameters of the proper motions of all sources in the field, derived from differences of the positions in the two epochs. Note that the position of the jet changes slightly between the epochs by about 6 mas in each coordinate $(\sim 3 \sigma)$. We do not attach any significance to this shift. If we aligned the jet positions, the proper motions listed in Table 2 would change by about 2 mas $\mathrm{yr}^{-1}$. This amount is insignificant and does not change any of our conclusions.

We note that the faint features that appear marginally detected at the $4 \sigma$ level in the images of the individual epochs (for example, some faint structures to the west of the central source in the 2006.42 image), do not appear in the difference image, since this is about $\sqrt{2}$ noisier than the individual images.

As already discussed by Rodríguez et al. (2005), the lobes first observed by Garay et al. (2003) break into several components. A more careful examination of the 2003 map in conjunction with the 2006 map shows that there are two other components present in the northern lobe (components N-4 and N-5; see Table 1 and Figure 2), as well as two additional field sources (sources D and E, see Table 1).

In this section we will discuss the sources individually when new information was found, and in the following section we focus on the search for variability and proper motions. Most of the interpretation is in the context of a jet and bipolar outflow. The P.A. of the jet is $-16 \pm 1^{\circ}$, whereas the P.A. of the CO lobes is about $-6^{\circ}$ and the P.A. of the line joining N-1 and S-1 is $-16^{\circ}$. We adopt a nominal angle for the jet outflow of $-16^{\circ}$.

\subsection{The Central Jet Source}

An important difference with respect to the analysis of Rodríguez et al. (2005) is that we recognize the presence of a compact source (source D) very close to the jet source (see Figure 2), about 1 ".2 to its NW. To obtain the parameters of 


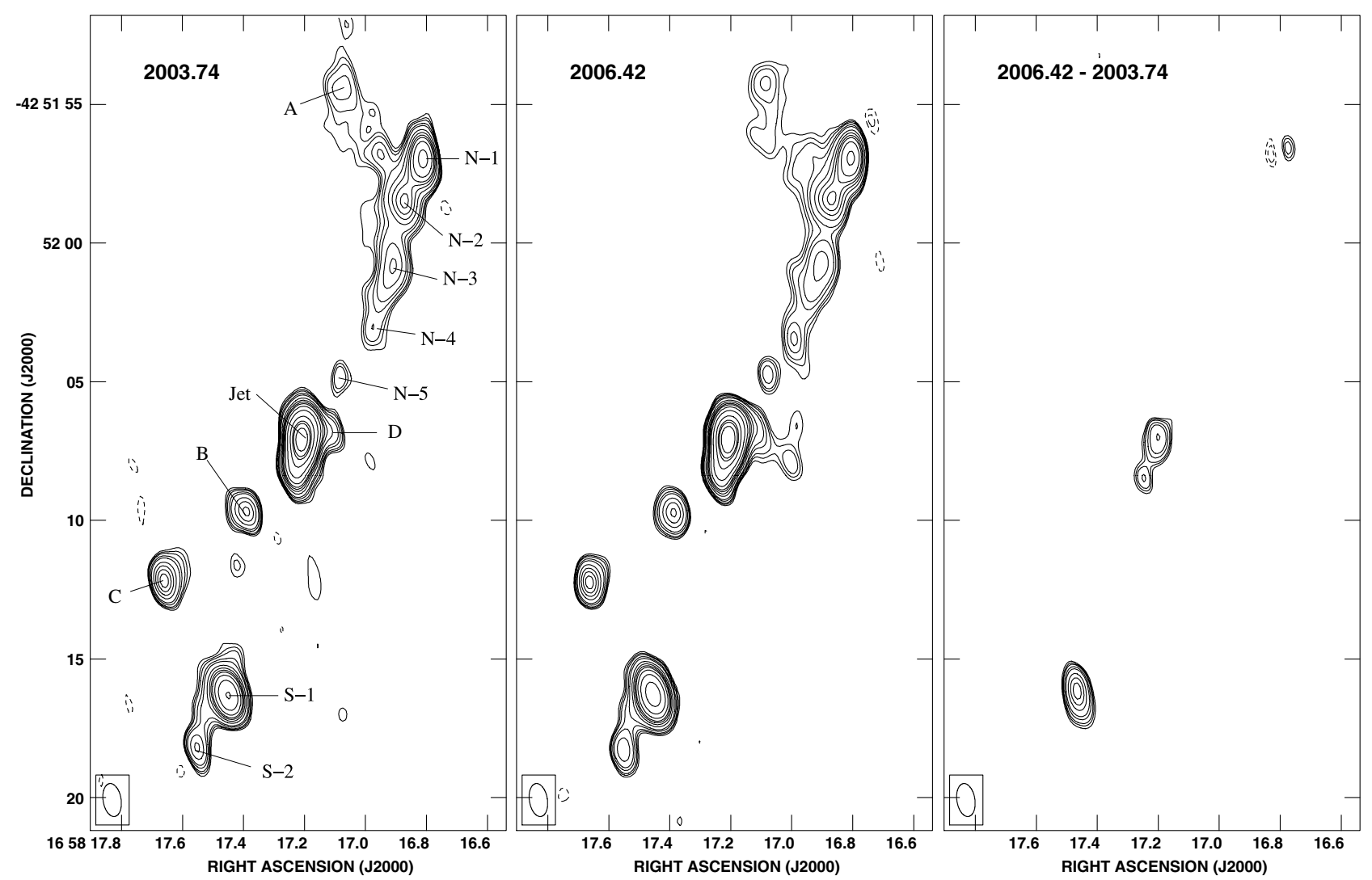

Figure 2. VLA contour images at $8.46 \mathrm{GHz}$ toward IRAS 16547-4247 for epochs 2003.74 (left) and 2006.42 (center), as well as the difference image (2006.422003.74). Contours are $-25,-20,-15,-10,-8,-6,-5,-4,4,5,6,8,10,15,20,25,30,40,60,100,140,160$, and 200 times $27 \mu \mathrm{Jy}_{\text {beam }}^{-1}$ for the 2003.74 and 2006.42 images (the average value of the rms noises of the two images) and $38 \mu \mathrm{Jy} \mathrm{beam}^{-1}$ for the difference image (the rms noise of this last image). The half power contour of the synthesized beams $\left(1^{\prime \prime} .20 \times 0^{\prime \prime} 65 ;\right.$ P.A. $\left.=9^{\circ}\right)$ is shown in the bottom-left corner of each panel. The individual sources are identified in the 2003.74 image.

the jet and of source D separately we fitted simultaneously two Gaussian ellipsoids to this region of the image. The jet parameters for the two epochs discussed are given in Table 1 . We note that the consideration of source D in the fitting procedure results in somewhat smaller deconvolved dimensions for the minor axis of the jet than that obtained in Rodríguez et al. (2005). We assume that the opening angle of the thermal jet is the angle subtended by the deconvolved minor axis at a distance of one-half the deconvolved major axis (Eislöffel et al. 2000). Using the average of the deconvolved angular dimensions given in Table 1, we then estimate the opening angle of the thermal jet to be $\sim 15^{\circ}$ (as opposed to the value of $\sim 25^{\circ}$ derived by Rodríguez et al.), indicating significant collimation in this massive protostar. This result suggests that jets from high-mass young stars can be as collimated as those found in lower-mass objects, where Hubble Space Telescope (HST) studies indicate opening angles in the range of tens of degrees in scales of tens of AU from the star (Ray et al. 2007). On larger physical scales the optical jets from low-mass young stars are known to show recollimation, resulting in opening angles of a few degrees (Ray et al. 2007). This recollimation is not evident in the thermal radio jets, which are usually detected only close to the star.

\subsection{The Northern Lobe}

As noted before, we identify two additional components ( $\mathrm{N}-4$ and $\mathrm{N}-5)$ in this lobe, indicating an almost continuous sequence of knots between the central jet and the outermost $\mathrm{N}-1$ component (see Figure 2). The sequence of five knots, N-1 to
$\mathrm{N}-5$, shows a gentle monotonic curvature that may be indicative of precession (see Section 5). Four of the five knots are resolved angularly, and it is interesting to note that all four have P.A.s consistent with $\sim 160^{\circ} \pm 10^{\circ}$. This result suggests that they are part of the northern outflow. This orientation does not seem to be valid for component $\mathrm{S}-1$, as discussed in the next subsection. The $\mathrm{N}-1$ and $\mathrm{N}-2$ continuum components are the only ones in the whole region that appear to be associated with class I methanol masers (Voronkov et al. 2006).

\subsection{The Southern Lobe}

Only the component S-1 is clearly resolved, but in contrast to the components of the northern lobe that have intrinsic P.A.s consistent with $\sim 160^{\circ} \pm 10^{\circ}$, the southern lobe's intrinsic P.A. is $\sim 40^{\circ}$. The misalignment between the intrinsic P.A. of S-1 and the outflow axis suggests that more than a part of the main jet body, S-1, could be its working surface (e.g., Chakrabarti 1988) to the south. As we will see below, the time variability of this component could support this interpretation. On the other hand, the presence of an additional component (S-2) downstream suggests that our interpretation of S-1 as a working surface may be incorrect, or that S-2 is an independent source associated with a star. The fact that $\mathrm{S}-2$ is unresolved is consistent with this interpretation.

\subsection{Source A}

This source is clearly resolved. It appears to be part of a diffuse region of emission that connects with the brighter 
Table 1

Observed $3.6 \mathrm{~cm}$ Parameters of Sources in the Field

\begin{tabular}{|c|c|c|c|c|}
\hline $\begin{array}{l}\text { Component } \\
\text { (Epoch) }\end{array}$ & $\begin{array}{l}\alpha(2000)^{\mathrm{a}} \\
\left(16^{\mathrm{h}} 58^{\mathrm{m}}\right)\end{array}$ & $\begin{array}{c}\delta(2000)^{\mathrm{a}} \\
\left(-42^{\circ}\right)\end{array}$ & $\begin{array}{c}\mathrm{S}_{v}^{\mathrm{b}} \\
(\mathrm{mJy})\end{array}$ & Deconvolved size ${ }^{c}$ \\
\hline $\mathrm{A}(2003)$ & $17.0597 \pm 0.0077$ & $51^{\prime} 55^{\prime \prime} .039 \pm 0 ! .240$ & $1.58 \pm 0.23$ & $4^{\prime \prime} .2 \pm 0^{\prime \prime} 6 \times 1^{\prime \prime} .2 \pm 0^{\prime \prime} .2 ; 9^{\circ} \pm 4^{\circ}$ \\
\hline$A(2006)$ & $17^{\mathrm{s}} .0641 \pm 0.0072$ & $51^{\prime} 55^{\prime \prime} \cdot 212 \pm 00^{\prime \prime} 205$ & $1.60 \pm 0.20$ & $4^{\prime \prime} .1 \pm 0^{\prime} .5 \times 1^{\prime \prime} .5 \pm 0^{\prime} .2 ; 4^{\circ} \pm 5^{\circ}$ \\
\hline N-1(2003) & $16.8165 \pm 0.0005$ & $51^{\prime} 57^{\prime \prime} .048 \pm 00^{\prime \prime} 011$ & $2.86 \pm 0.07$ & $1^{\prime \prime} .06 \pm 0^{\prime \prime} .04 \times 0^{\prime \prime} .31 \pm 0^{\prime} .05 ; 163^{\circ} \pm 3^{\circ}$ \\
\hline N-1(2006) & $5.8136 \pm 0.0004$ & $51^{\prime} 57^{\prime \prime} .060 \pm 0.009$ & $2.85 \pm 0.06$ & $1^{\prime \prime} .15 \pm 0^{\prime \prime} .03 \times 0^{\prime \prime} .23 \pm 0^{\prime \prime} .08 ; 156^{\circ} \pm 2^{\circ}$ \\
\hline N-2(2003) & $16.8706 \pm 0.0015$ & $51^{\prime} 58^{\prime \prime} .339 \pm 0^{\prime \prime} 028$ & $2.35 \pm 0.10$ & $1^{\prime \prime} .65 \pm 0^{\prime \prime} .09 \times 0^{\prime \prime} .80 \pm 0^{\prime \prime} .08 ; 162^{\circ} \pm 5^{\circ}$ \\
\hline N-2(2006) & $16^{\mathrm{s}} .8669 \pm 0.0011$ & $51^{\prime} 58^{\prime \prime} .249 \pm 0.0^{\prime \prime} 019$ & $2.24 \pm 0.07$ & $1^{\prime \prime} .47 \pm 0^{\prime \prime} .07 \times 0^{\prime \prime} .76 \pm 0^{\prime \prime} .07 ; 151^{\circ} \pm 5^{\circ}$ \\
\hline & $=0.0021$ & $52^{\prime} 00^{\prime \prime} 776 \pm 0^{\prime \prime}$. & $1.82 \pm 0.12$ & $2^{\prime \prime} .52 \pm 0^{\prime \prime} .17 \times 0^{\prime \prime} .62 \pm 0^{\prime \prime} .10 ; 168^{\circ} \pm 3^{\circ}$ \\
\hline N-3(2006) & $16^{\mathrm{s}} .9114 \pm 0.0022$ & $52^{\prime} 00^{\prime \prime} 921 \pm 0^{\prime \prime} 052$ & $1.76 \pm 0.10$ & $2^{\prime \prime} .52 \pm 0^{\prime \prime} .14 \times 0^{\prime \prime} .78 \pm 0^{\prime} .09 ; 162^{\circ} \pm 3^{\circ}$ \\
\hline $\mathrm{E}(2003)$ & $18.2348 \pm 0.0080$ & $52^{\prime} 02^{\prime \prime} 183 \pm 00^{\prime \prime} 115$ & $0.16 \pm 0.05$ & $\leqslant 1^{\prime \prime} \cdot 2^{\mathrm{d}}$ \\
\hline $\mathrm{E}(2006)$ & $18.2128 \pm 0.0044$ & $52^{\prime} 02^{\prime \prime} .319 \pm$ & $0.12 \pm 0.04$ & $\leqslant 1^{\prime \prime} 1^{\mathrm{d}}$ \\
\hline & & & $0.87 \pm 0.14$ & $2^{\prime \prime} .66 \pm 0^{\prime \prime} .43 \times 0^{\prime \prime} .88 \pm 0^{\prime} .27 ; 171^{\circ} \pm 9^{\circ}$ \\
\hline N-4(2006) & $16^{\mathrm{s}} .9851 \pm 0.0033$ & $52^{\prime} 03^{\prime \prime} \cdot 286 \pm 0.0^{\prime \prime} 092$ & $0.86 \pm 0.09$ & $2^{\prime \prime} .40 \pm 0^{\prime \prime} .25 \times 0^{\prime \prime} .71 \pm 0^{\prime \prime} .16 ; 170^{\circ} \pm 5^{\circ}$ \\
\hline $\mathrm{N}-5(2003)$ & $17^{\mathrm{s}} .0764 \pm 0.0053$ & $52^{\prime} 04^{\prime \prime} 881 \pm 0.079$ & $0.27 \pm 0.06$ & \\
\hline N-5(2006) & $17^{\mathrm{s}} .0715 \pm 0.0024$ & $52^{\prime} 04^{\prime \prime} .737 \pm 0^{\prime \prime} 046$ & $0.30 \pm 0.04$ & $\leqslant 0^{\prime \prime} \cdot 7^{\mathrm{d}}$ \\
\hline $\mathrm{D}(2003)$ & $17.1265 \pm 0.0016$ & $52^{\prime} 06^{\prime \prime} 840 \pm 00^{\prime} 031$ & $0.50 \pm 0.03$ & $\leqslant 0^{\prime \prime} 7^{\mathrm{d}}$ \\
\hline $\mathrm{D}(2006)$ & $17^{\mathrm{s}} .1211 \pm 0.0012$ & $52^{\prime} 06^{\prime \prime} 833 \pm 0.023$ & $0.48 \pm 0.02$ & $\leqslant 0^{\prime \prime} \cdot 6^{\mathrm{d}}$ \\
\hline Jet(2003) & $17.2097 \pm 0.0001$ & $52^{\prime} 07^{\prime \prime} .142 \pm 0^{\prime \prime} 003$ & $10.4 \pm 0.1$ & $1^{\prime \prime} .21 \pm 0^{\prime \prime} 01 \times 0.0^{\prime} 15 \pm 0.03$ \\
\hline Jet(2006) & $17.2093 \pm 0.0001$ & $52^{\prime} 07^{\prime \prime} .150 \pm 0^{\prime} .002$ & $11.3 \pm 0.1$ & $1^{\prime \prime} .20 \pm 0^{\prime} .01 \times 0^{\prime \prime} .14 \pm 0^{\prime} .02 ; 163^{\circ} \pm 1^{\circ}$ \\
\hline $\mathrm{B}(2003)$ & $17^{\$} .3946 \pm 0.0013$ & $52^{\prime} 09^{\prime \prime} 675 \pm 0.0^{\prime \prime} 019$ & $0.81 \pm 0.06$ & \\
\hline$B(2006)$ & $17.3904 \pm 0.0009$ & $52^{\prime} 09^{\prime \prime} .732 \pm 0^{\prime \prime} .015$ & $0.85 \pm 0.04$ & $\leqslant 0^{\prime \prime} \cdot 5^{\mathrm{d}}$ \\
\hline$C(2003)$ & $17^{\mathrm{s}} .6549 \pm 0.0010$ & $52^{\prime} 12^{\prime \prime} 150 \pm 0^{\prime \prime} 018$ & $1.10 \pm 0.06$ & $\leqslant 0^{\prime \prime} 6^{\mathrm{d}}$ \\
\hline $\mathrm{C}(2006)$ & $17.6603 \pm 0.0007$ & $52^{\prime} 12^{\prime \prime} .221 \pm 0^{\prime \prime} 012$ & $0.97 \pm 0.04$ & $\leqslant 0^{\prime \prime} .5^{\mathrm{d}}$ \\
\hline S-1(2003) & $17.4519 \pm 0.0004$ & $52^{\prime} 16^{\prime \prime} \cdot 301 \pm 00^{\prime \prime} 006$ & $3.65 \pm 0.06$ & $0^{\prime \prime} .59 \pm 0^{\prime} .04 \times 0^{\prime \prime} .40 \pm 0^{\prime \prime} .05 ; 51^{\circ} \pm 9^{\circ}$ \\
\hline S-1(2006) & $17.4556 \pm 0.0002$ & $52^{\prime} 16^{\prime \prime} \cdot 284 \pm 00^{\prime \prime} 003$ & $4.97 \pm 0.05$ & $0^{\prime \prime} .66 \pm 0^{\prime} .02 \times 0^{\prime \prime} .38 \pm 0^{\prime} .02 ; 35^{\circ} \pm 4^{\circ}$ \\
\hline S-2(2003) & $17.5481 \pm 0.0013$ & $52^{\prime} 18^{\prime \prime} .113 \pm 00^{\prime \prime} 031$ & $0.63 \pm 0.06$ & \\
\hline S-2(2006) & $17.5473 \pm 0.0011$ & $52^{\prime} 18^{\prime \prime} .210 \pm 0^{\prime \prime} 026$ & $0.69 \pm 0.05$ & $\leqslant 0^{\prime \prime} 8^{\mathrm{d}}$ \\
\hline
\end{tabular}

Notes.

a Peak position. Right ascension $(\alpha)$ given in hours, minutes, and seconds, and declination $(\delta)$, given in degrees, arcmins, and arcsecs. The errors given are formal statistical errors. The absolute positional error of the images are estimated to be 0.2 in right ascension and 0.5 in declination. The sources are listed in order of decreasing declination.

b Total flux density. The errors given are formal statistical errors. The absolute flux-density error is estimated to be on the order of $10 \%$.

${ }^{c}$ Deconvolved dimensions of the source: FWHM major axis $\times$ FWHM minor axis; P.A. of major axis.

${ }^{\mathrm{d}}$ Unresolved.

component of the northern lobe. Source A could actually be a part of the outflow, either if the outflow is less collimated and the ionized gas preferentially highlights regions where the flow is interacting with a dense ambient material or if source $\mathrm{A}$ is a part of the northern flow being deflected to the east by interaction with a dense clump of gas. The second possibility is interesting and it may be related to the fact that components $\mathrm{N}-1$ and $\mathrm{N}-2$ are the only those in the region showing methanol maser emission. It is possible that the methanol maser emission is being stimulated by the deflection of the jet, as it interacts with $\mathrm{N}-1$ and $\mathrm{N}-2$. Finally, the position of source A could be understood if the outflow has a precession (see discussion in Section 5). High-angular-resolution observations of a tracer of dense molecular gas are needed to advance our understanding of this source.

\subsection{Source D}

Source D is the faint source located $1^{\prime \prime} .2$ to the northwest of the jet, and first identified as an independent source here. It is unresolved angularly and may probably to trace an independent star. In the 2006.42 image, it shows a faint extension to the west.

\subsection{Source E}

Source E is a barely detected source located about $10^{\prime \prime}$ to the east of the core of IRAS 16547-4247. It is located outside of the region shown in Figure 2, and is unresolved in angular size. A contour image of this source is shown in Figure 3. The a priori probability of finding a $3.6 \mathrm{~cm}$ background source with a flux density of $\sim 0.14 \mathrm{mJy}$ (the average flux density of the two epochs) in a solid angle of $20^{\prime \prime} \times 20^{\prime \prime}$ is only $\sim 0.003$ (Windhorst et al. 1993). We conclude that this radio source most probably traces a young star embedded in this region.

\section{SEARCH FOR VARIATION AND PROPER MOTIONS}

Analysis of Figure 2 and Table 1 indicates that significant flux-density or morphological variations are observed only in three sources: the jet source, $\mathrm{N}-1$, and $\mathrm{S}-1$. This is not unexpected, since these are the three brightest sources in the field and small variations in position or flux density are not evident in weaker sources where the signal-to-noise ratio is much smaller. In the case of $\mathrm{N}-1$ the variation is only in position since the flux density remained constant and the flux-density variations are evident only for the jet source and S-1. We will discuss our interpretation of the observed variabilities and proper motions in the following subsections.

\subsection{The Central Jet Source and Source S-1}

The jet source seems to have increased its flux density by about $10 \%$ between the two epochs (see Table 1 and 


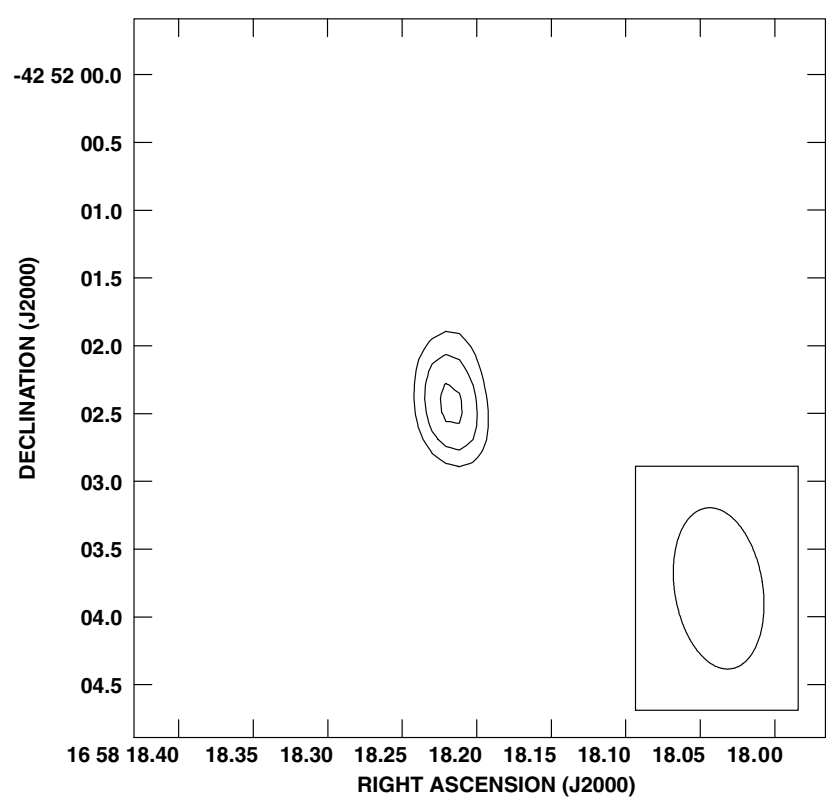

Figure 3. VLA contour images at $8.46 \mathrm{GHz}$ of the source E, made from the average of both epochs (2003.74 and 2006.42). Contours are $-4,4,5$, and 6 times $18 \mu \mathrm{Jy}_{\text {beam }}^{-1}$, the rms noise of the image. The half-power contour of the synthesized beam is as in Figure 2.

Figure 1). This difference is consistent with the fact that both thermal jets (Rodríguez et al. 2001; Galván-Madrid et al. 2004) and $\mathrm{HH}$ knots (Rodríguez et al. 2000) can show small but statistically significant flux-density variations on scales of years or even months. The difference image (Figure 4) suggests that the increase in emission comes from two discrete positions. One increase (of about $0.8 \mathrm{mJy}$ ) is unresolved and associated with the central source and we attribute it to an increase in mass loss at the core of the jet. The second increase (of about $0.4 \mathrm{mJy}$ ) comes from an unresolved component clearly displaced to the SE, and located at $\alpha(2000)=16^{\mathrm{h}} 58^{\mathrm{m}} 17^{\mathrm{s}} .2446 \pm 0.0033 ; \delta(2000)=$ $-42^{\circ} 52^{\prime} 08^{\prime \prime} .354 \pm 00^{\prime \prime} 067$, at $1^{\prime \prime} .3$ from the center of the jet. The $\mathrm{OH}$ maser emission detected by Caswell (1998) is close to the unresolved component associated with this second increase. We can think of three interpretations for this localized fluxdensity increase. One is that we are observing a time-variable source powered by an independent star. There are two arguments against this interpretation: the first is that the sources in this region in general show little variation (see Table 1) and the second is that the source lies exactly in the path of the outflow, suggesting a relation with the jet. This latter fact suggests a second interpretation: we are seeing a discrete, new ejecta from the jet. This interpretation, on its turn, has several difficulties. The first is that, while a similar phenomenon was observed and monitored in the jet associated with $\mathrm{HH}$ 80-81 (Martí et al. 1995, 1998), in this source the ejection was clearly bipolar, while in IRAS 16547-4247 we would have to consider a monopolar ejection. The second problem is related to the large velocity required for the ejection to move over 1".3 in 2.68 years or less, that at a distance of $2.9 \mathrm{kpc}$ implies the unlikely velocity of $\sim 10^{4} \mathrm{~km} \mathrm{~s}^{-1}$ or more. Finally, a third possibility is that we are observing the brightening of the jet flow as it interacts with dense gas in its path. Observations of a higher angular resolution are required to disentangle the nature of the variations seen in the jet source. Unfortunately, the present observations are the best that can be done now and possibly for decades (until completion of the Square Kilometer Array). The source can be observed, but with a lower angular resolution, with ATCA (as already done) but it cannot be observed from the latitude of the future e-MERLIN. Finally, the emission is thermal and not easily detectable with VLBI arrays.

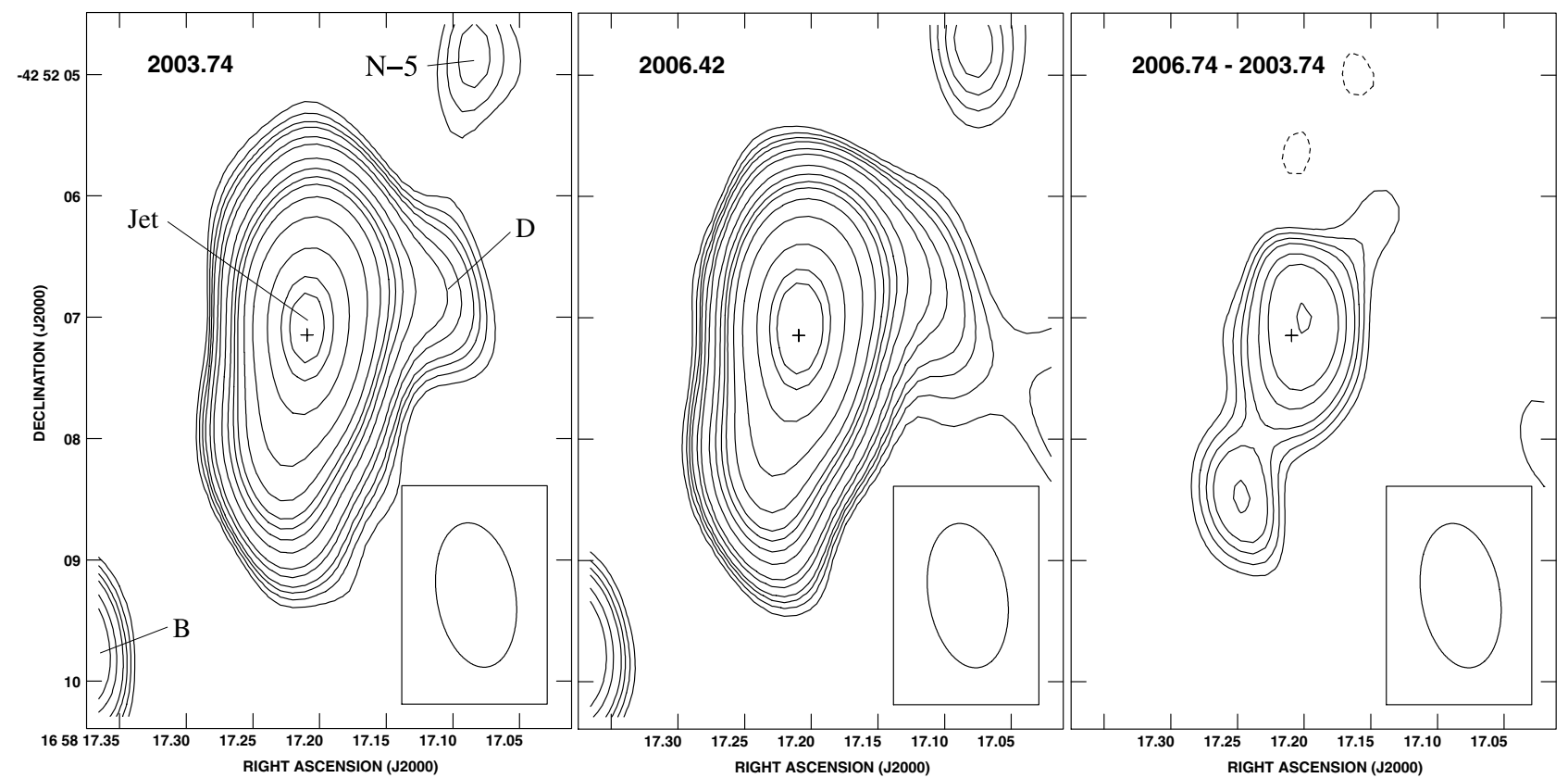

Figure 4. VLA contour images at $8.46 \mathrm{GHz}$ toward the jet in IRAS $16547-4247$ for epochs 2003.74 (left) and 2006.42 (center), as well as the difference image (2006.42-2003.74). Contours are $-25,-20,-15,-10,-8,-6,-5,-4,4,5,6,8,10,15,20,25,30,40,60,100,140,160$, and 200 times $27 \mu \mathrm{Jy}$ beam ${ }^{-1}$ for the 2003.74 and 2006.42 images and $38 \mu \mathrm{Jy}_{\text {beam }}{ }^{-1}$ for the difference image. The half-power contour of the synthesized beams $\left(1^{\prime \prime} .20 \times 0.0^{\prime \prime} 65 ;\right.$ P.A. $\left.=99^{\circ}\right)$ is shown in the bottom-right corner. The cross marks the peak position of the jet as determined from the average of the two positions given in Table 1 . Individual sources are identified in the 2003.74 image. 
Table 2

Proper Motions of Sources

\begin{tabular}{|c|c|c|c|c|c|c|c|c|c|}
\hline Source & $\begin{array}{l}D^{\mathrm{a}} \\
\left({ }^{\prime \prime}\right)\end{array}$ & $\begin{array}{c}\text { P.A. }(\mathrm{O})^{\mathrm{b}} \\
\left(^{\circ}\right)\end{array}$ & $\begin{array}{c}\text { P.A. }(\mathrm{P})^{\mathrm{c}} \\
\left({ }^{\circ}\right)\end{array}$ & $\begin{array}{c}\mu(\alpha)^{\mathrm{d}} \\
\left(\operatorname{mas} \mathrm{yr}^{-1}\right)\end{array}$ & 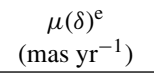 & $\begin{array}{c}\mu^{\mathrm{f}} \\
\left(\operatorname{mas~yr}^{-1}\right)\end{array}$ & $\begin{array}{c}\text { P.A. }(\mu)^{\mathrm{g}} \\
\left(^{\circ}\right)\end{array}$ & 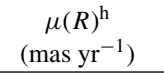 & 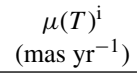 \\
\hline A & 12.215 & -7.76 & -15 & $18 \pm 43$ & $-65 \pm 118$ & $67 \pm 114$ & $164 \pm 97$ & $-67 \pm 114$ & $0 \pm 53$ \\
\hline $\mathrm{N}-1$ & 10.981 & -23.19 & -18 & $-12 \pm 3$ & $-4 \pm 5$ & $13 \pm 3$ & $-111 \pm 14$ & $-1 \pm 5$ & $-13 \pm 3$ \\
\hline $\mathrm{N}-2$ & 9.560 & -22.96 & -21 & $-15 \pm 8$ & $34 \pm 13$ & $37 \pm 12$ & $-24 \pm 19$ & $36 \pm 12$ & $-5 \pm 8$ \\
\hline $\mathrm{N}-3$ & 7.147 & -27.04 & -27 & $-11 \pm 12$ & $-54 \pm 30$ & $55 \pm 29$ & $-168 \pm 30$ & $-49 \pm 29$ & $-26 \pm 15$ \\
\hline $\mathrm{E}$ & 12.316 & 66.26 & -15 & $-90 \pm 37$ & $-51 \pm 58$ & $104 \pm 43$ & $-119 \pm 24$ & $-24 \pm 57$ & $-101 \pm 39$ \\
\hline $\mathrm{N}-4$ & 5.239 & -30.98 & -31 & $85 \pm 28$ & $-237 \pm 70$ & $252 \pm 67$ & $160 \pm 15$ & $-251 \pm 68$ & $16 \pm 34$ \\
\hline $\mathrm{N}-5$ & 2.695 & -32.96 & -37 & $-20 \pm 24$ & $54 \pm 34$ & $57 \pm 33$ & $-21 \pm 33$ & $57 \pm 33$ & $-5 \pm 25$ \\
\hline $\mathrm{D}$ & 0.964 & -71.73 & -41 & $-22 \pm 8$ & $3 \pm 14$ & $22 \pm 8$ & $-83 \pm 21$ & $9 \pm 14$ & $-21 \pm 9$ \\
\hline Jet & 0.000 & 0.00 & -43 & $-2 \pm 1$ & $-3 \pm 1$ & $3 \pm 1$ & $-151 \pm 20$ & $-2 \pm 1$ & $-2 \pm 1$ \\
\hline B & 3.248 & 141.24 & 144 & $-17 \pm 6$ & $-21 \pm 9$ & $27 \pm 8$ & $-141 \pm 17$ & $17 \pm 9$ & $22 \pm 7$ \\
\hline $\mathrm{C}$ & 7.004 & 135.65 & 153 & $22 \pm 5$ & $-26 \pm 8$ & $35 \pm 7$ & $140 \pm 12$ & $31 \pm 8$ & $-16 \pm 5$ \\
\hline S-1 & 9.538 & 163.79 & 159 & $15 \pm 2$ & $6 \pm 3$ & $16 \pm 2$ & $67 \pm 7$ & $-3 \pm 2$ & $-16 \pm 2$ \\
\hline S-2 & 11.585 & 161.26 & 164 & $-3 \pm 7$ & $-36 \pm 15$ & $36 \pm 15$ & $-175 \pm 24$ & $35 \pm 15$ & $11 \pm 8$ \\
\hline
\end{tabular}

Notes.

${ }^{a}$ Displacement with respect to the jet in arcsec.

$\mathrm{b}$ Observed P.A. with respect to the jet in degrees.

${ }^{c}$ Predicted P.A. in the precession model.

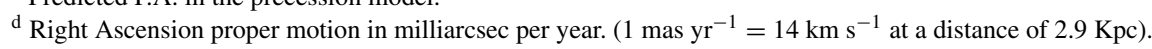

e Declination proper motion in milliarcsec per year.

$\mathrm{f}$ Total proper motion in milliarcsec per year.

P.A. of the total proper motion in degrees.

${ }^{\mathrm{h}}$ Radial proper motion with respect to an axis with P.A. $=-16^{\circ}$, in milliarcsec per year. A negative sign corresponds to motions approaching the jet.

${ }^{\mathrm{i}}$ Transversal proper motion with respect to an axis with P.A. $=-16^{\circ}$, in milliarcsec per year. A positive sign corresponds to counterclockwise motion.

${ }^{j}$ Note that if the maps were aligned to make the position of the jet fixed, the proper motions of the other components would

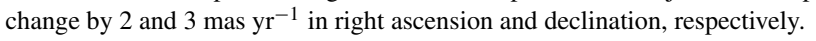

The source S-1 is the one with the largest flux-density variation, with an increase of $\sim 40 \%$ (see Figure 1 and Table 1) between the two epochs. We believe that this large increase is related to our suggestion that S-1 may be a working surface of the jet, where kinetic energy is rapidly being dissipated and changes are expected.

\subsection{Lack of Proper Motions Along the Jet Axis}

The proper motions of the prominent components $\mathrm{N}-1$ and $\mathrm{S}-1$ along the nominal jet axis of $-16^{\circ}$ are $-2 \pm 5$ and $-3 \pm$ 2 mas $\mathrm{yr}^{-1}$, respectively. These motions correspond to inward motions of 28 and $42 \mathrm{~km} \mathrm{~s}^{-1}$, respectively, and are not statistically significant. The weighted average radial motion of

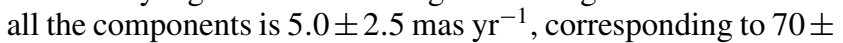
$40 \mathrm{~km} \mathrm{~s}^{-1}$. We adopt a conservative $4 \sigma$ upper limit on the proper motion of $160 \mathrm{~km} \mathrm{~s}^{-1}$. This upper limit is not very stringent, but certainly indicates that the IRAS 16547-4247 lobes are not moving as fast as the knots observed in the jets associated with the massive young stars $\mathrm{HH}$ 80-81 and Cep A HW2, where velocities in the plane of the sky of $\sim 500 \mathrm{~km} \mathrm{~s}^{-1}$ have been reported (Martí et al. 1998; Curiel et al. 2006).

\subsection{Proper Motions in the Direction Transverse to the Jet Axis}

The only components that show transverse motions to the nominal jet direction of -16 degrees above the $4 \sigma$ level are $\mathrm{N}-1$ and S-1 (see Table 2). These motions are $-13 \pm 3$ and $-16 \pm$ 2 mas $\mathrm{yr}^{-1}$, corresponding to clockwise transverse motions of $180 \pm 40$ and $220 \pm 30 \mathrm{~km} \mathrm{~s}^{-1}$, respectively. The discovery of transverse (clockwise) motion, with no radial motion, was unexpected.
The total flux density of the component N-1 hardly changed, and the difference images shown in Figures 2 and 5 clearly show the negative-positive residuals characteristic of a moving source with constant flux density. In contrast, the negativepositive signature of proper motion is not present in the case of S-1 (see Figures 2 and 6) because during the same period there was the strong brightening previously discussed that dominated the difference image.

\section{A PRECESSION MODEL FOR IRAS 16547-4247}

We believe that it may be possible to understand the distribution of almost all the identified sources as well as the clockwise precession as the result of ejecta from a precessing jet of the stellar origin. The gently curved and antisymmetric distribution of the radio components strongly suggests such a model. The basic idea is that the jet itself is not observed directly (because of the lack of radial motion in the outflow). Rather the observed sources are the result of the jet interacting with the ambient medium. We describe this model in simple empirical terms. We assume that the axis of symmetry is along the north-south direction and that the P.A. (measured east of north) of the precession axis is given by

$$
\theta \sim \theta_{m} \sin (\omega t)-\theta_{0}
$$

where $\theta_{m}$ is the amplitude of precession, $\omega$ is the precession rate and $\theta_{0}$ is the P.A. at $t=0$. We assume that the timescale of the observable outflow phenomenon is much shorter than the precession period. Hence, over a short period the precession P.A. changes linearly,

$$
\theta \simeq \theta_{m} \omega t-\theta_{0}=\beta t-\theta_{0} .
$$



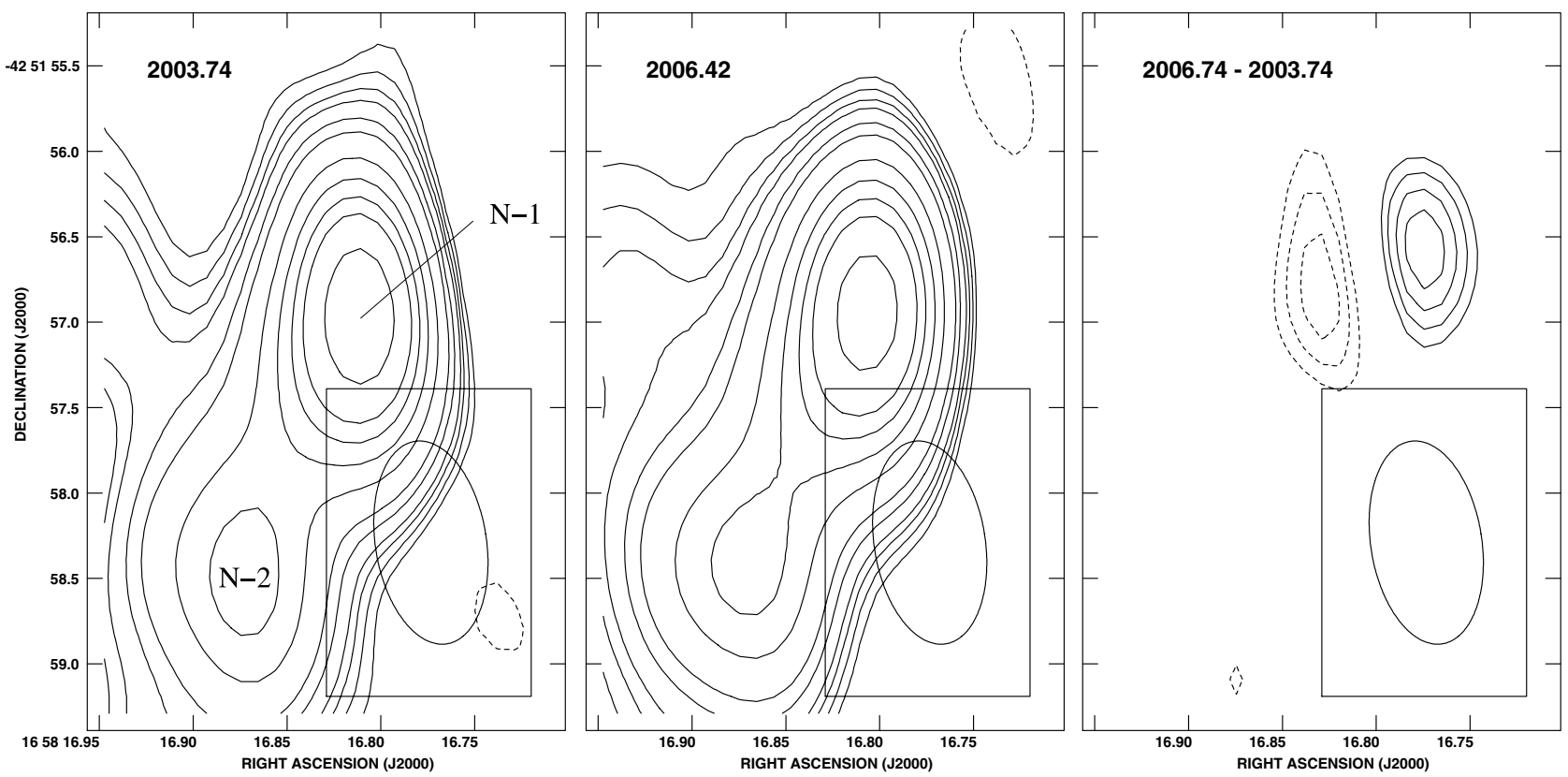

Figure 5. VLA contour images at $8.46 \mathrm{GHz}$ toward the component N-1 in IRAS $16547-4247$ for epochs 2003.74 (left) and 2006.42 (center), as well as the difference image (2006.42-2003.74). Contours are $-25,-20,-15,-10,-8,-6,-5,-4,4,5,6,8,10,15,20,25,30,40,60,100,140,160$, and 200 times $27 \mu \mathrm{Jy}_{\text {beam }}{ }^{-1}$ for the 2003.74 and 2006.42 images and $38 \mu \mathrm{Jy}_{\text {beam }}{ }^{-1}$ for the difference image. The half-power contour of the synthesized beams $\left(1^{\prime \prime} .20 \times 00^{\prime \prime} 65 ;\right.$ P.A. $\left.=9^{\circ}\right)$ is shown in the bottom-right corner. Individual sources are identified in the 2003.74 image.
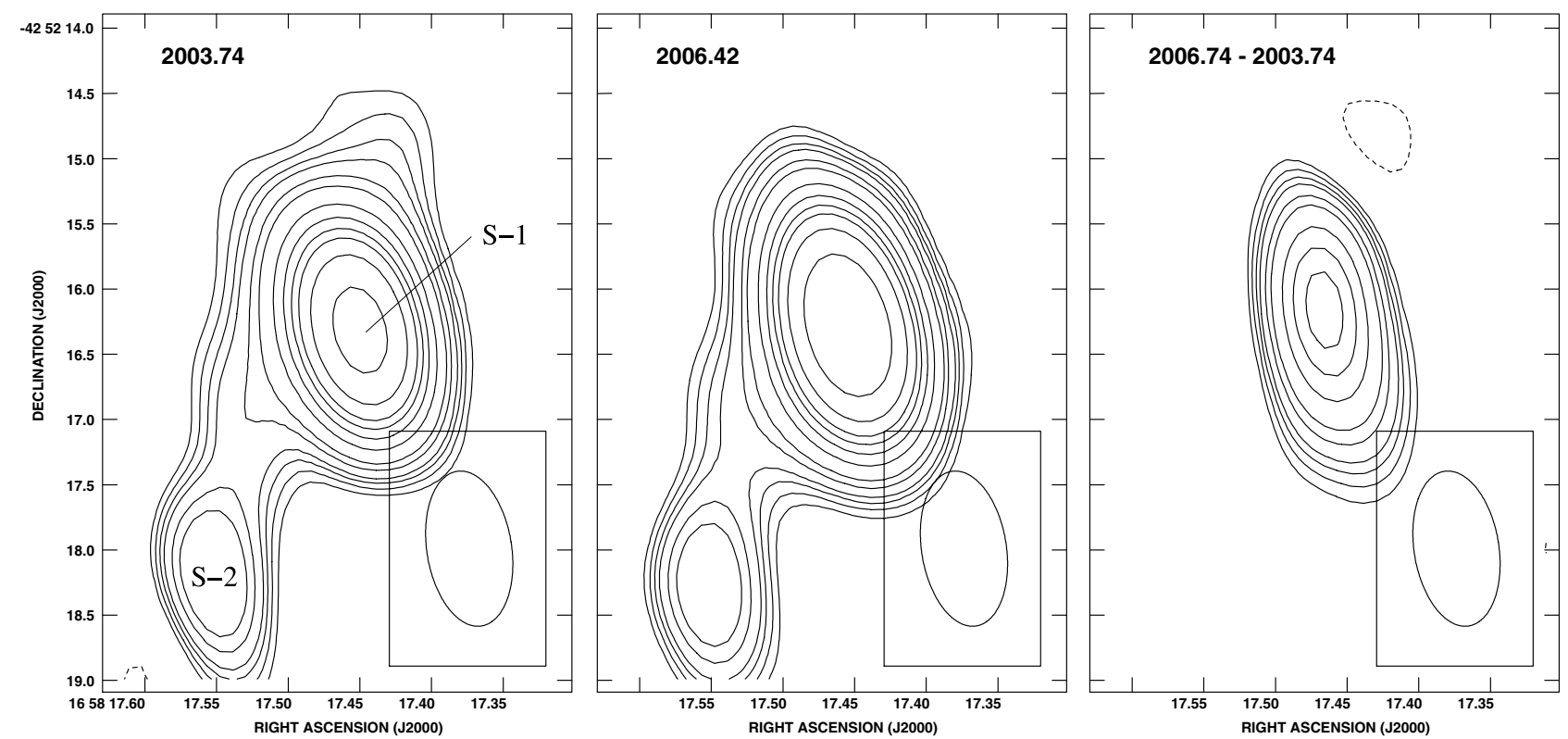

Figure 6. VLA contour images at $8.46 \mathrm{GHz}$ toward the component S-1 in IRAS 16547-4247 for epochs 2003.74 (left) and 2006.42 (center), as well as the difference image (2006.42-2003.74). Contours are $-25,-20,-15,-10,-8,-6,-5,-4,4,5,6,8,10,15,20,25,30,40,60,100,140,160$, and 200 times $27 \mu \mathrm{Jy}_{\text {beam }}-1$ for the 2003.74 and 2006.42 images and $38 \mu \mathrm{Jy}$ beam $^{-1}$ for the difference image. The half-power contour of the synthesized beams $\left(1^{\prime \prime} .20 \times 0^{\prime \prime} 65 ;\right.$ P.A. $\left.=9^{\circ}\right)$ is shown in the bottom-right corner. Individual sources are identified in the 2003.74 image.

The coordinates in the plane of the sky at the time of our observations, $t$, for the location of gas ejected from the star at time $t_{e}$ will be

$$
\begin{aligned}
& x(t)=r(t) \sin \theta_{e} \\
& y(t)=r(t) \cos \theta_{e},
\end{aligned}
$$

where $\theta_{e}$ is the precession angle at the time of emission, and $r$ is the radial position of the ejecta, $r=v\left(t-t_{e}\right)$. We assume that the ejection velocity, $v$, is constant. Since $t-t_{e}=r / v$ we obtain

$$
\begin{aligned}
& x(t)=r \sin \left(\frac{\beta r}{v}-\theta_{0}\right) \\
& y(t)=r \cos \left(\frac{\beta r}{v}-\theta_{0}\right) .
\end{aligned}
$$

The prediction of this simple model is that $\theta$ is linearly related to $r$, that is 


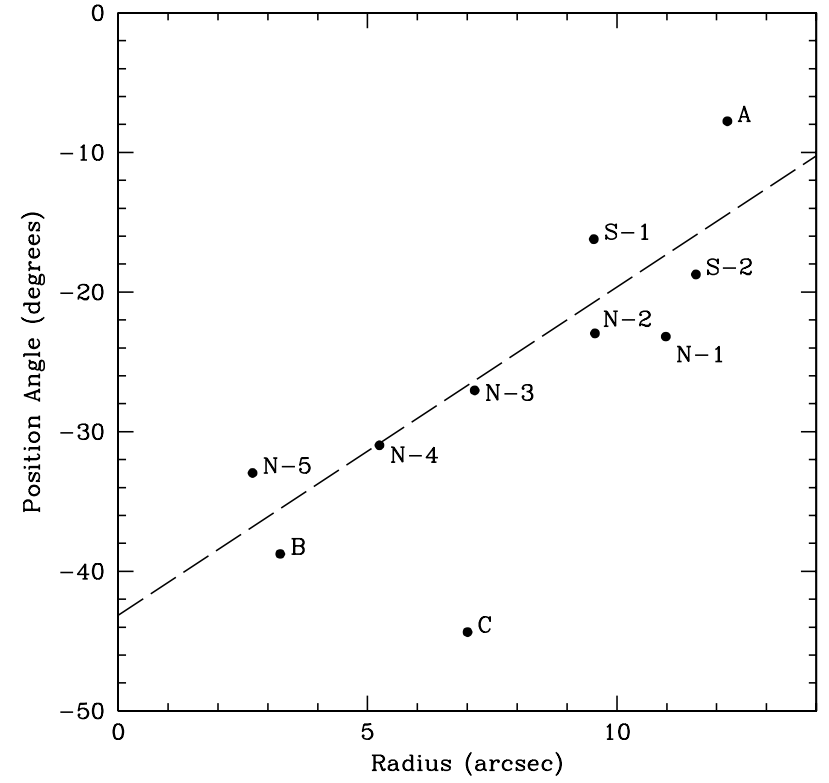

Figure 7. P.A. of the jet components as a function of radial offset from the jet center. The components south of the jet have been folded by $180^{\circ}$ in this figure. The dashed line is the linear least-squares fit to the components, with the exception of source $\mathrm{C}$, which is taken to be an independent star.

$$
\theta(r)=\beta r / v-\theta_{0} .
$$

Figure 7 shows the P.A. of each component plotted versus its radial offset from the center of the jet component, which we identify as the origin. Source $\mathrm{C}$ deviates from the straight-line fit to the data by about $19^{\circ}$. We assume that it is not a part of the jet, but a separate entity from the jet, perhaps evidence of another nearby star. The straight-line fit of Equation (7) to the remaining nine sources gives parameters, $\theta_{0}=-43^{\circ} \pm 4^{\circ}$ (the current P.A. of the jet), and $\alpha=\beta / v=2.3 \pm 0.4 \mathrm{deg}_{\operatorname{arcsec}}{ }^{-1}$. For a distance of $2.9 \mathrm{kpc}$ the value of $\alpha$ in the source frame is $9.2 \times 10^{-19}$ radians $\mathrm{cm}^{-1}$ or 2.9 radians $\mathrm{pc}^{-1}$. Since the jet seems to be precessing linearly with time, we assume that the precession period is much greater than the timescale of the flow. Note that we cannot solve separately for the precession rate and the ejection velocity. The shape of the jet at the current epoch, as determined by Equations (5) and (6), is shown in Figure 8.

As noted before, only two sources have significant proper motions, $\mathrm{N}-1$ and $\mathrm{S}-1$. Their motions are both in the transverse direction to the direction of the jet, whereas their motions along the jet are insignificant (see Table 2). Hence, we assume that the sources are caused by the interaction of the jet and clumps of ambient material as the jet sweeps across the clumps. In this model, the clumps will have apparent transverse velocities given by

$$
v_{T}=r \beta=r \alpha v,
$$

which, with the observed value of $\alpha$, becomes

$$
v_{T}=0.42 v\left(r / 10^{\prime \prime}\right) .
$$

The proper-motion measurements of all the sources are listed in Table 2. We believe that the apparent motions of source $\mathrm{C}$ could be due to the presence of an additional, time-variable component to the northwest of the main component of source $\mathrm{C}$ (see Figure 2). Source N-4 shows proper motions that are slightly below $4 \sigma$ (see Table 2). However, these marginal proper motions are against the expected flow of the jet and, if real, are probably due to a "Christmas tree" effect more that to a real motion.

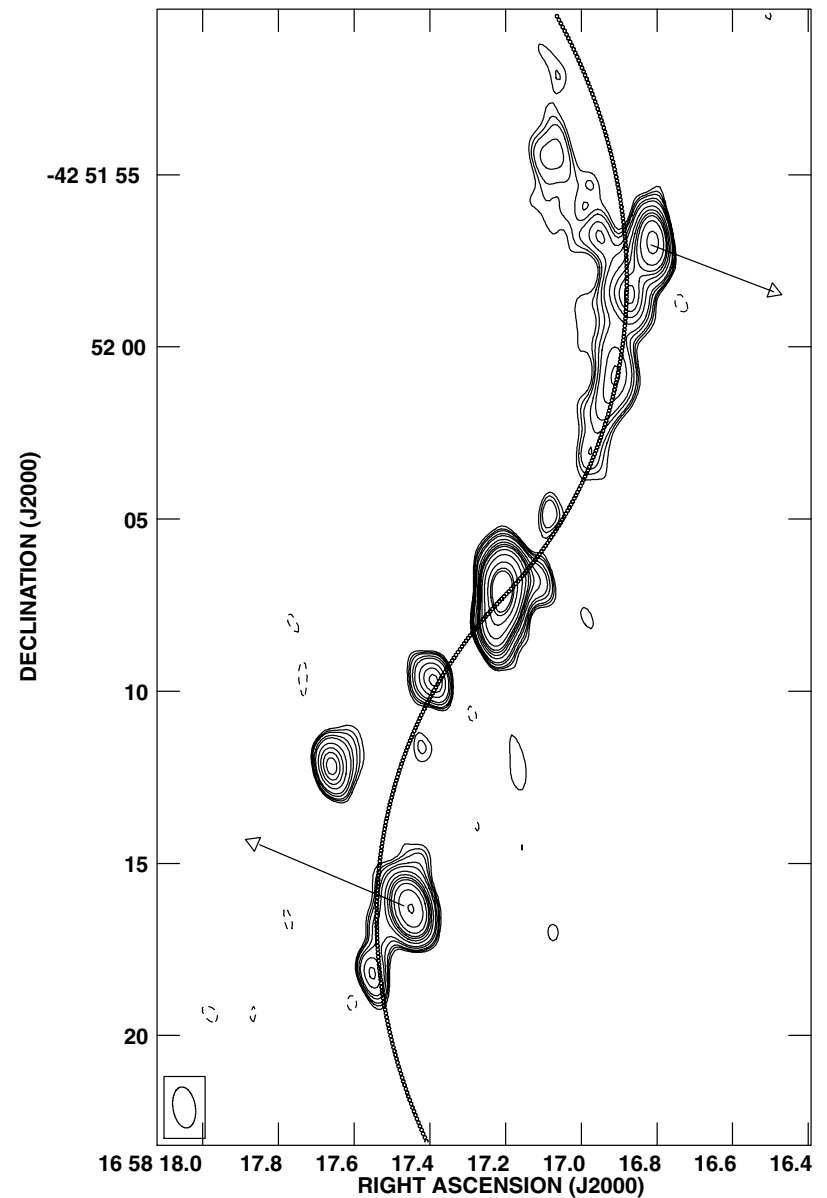

Figure 8. VLA contour image at $8.46 \mathrm{GHz}$ toward IRAS $16547-4247$ for epoch 2003.74. Contours and beam are as in Figure 2. The solid line indicates the position of the spiral model discussed in the text. The arrows indicate the proper motions of components $\mathrm{N}-1$ and $\mathrm{S}-1$ for a period of 300 years.

The velocity vectors of S-1 and $\mathrm{N}-1$ are nearly transverse to the jet direction, and agree in direction with the sense of precession of the jet (see Figure 8). These velocities are 180 and $220 \mathrm{~km} \mathrm{~s}^{-1}$, respectively. Since N-1 and S-1 both have about the same radial distance, we cannot confirm the expected linear trend in transverse velocity with a radius predicted by Equation (8). However, with these two data points we estimate from Equation (9) that the ejection velocity is $490 \pm 80 \mathrm{~km} \mathrm{~s}^{-1}$. If we assume that we are indeed dealing with a working surface, the lack of proper motions $\left(\leqslant 160 \mathrm{~km} \mathrm{~s}^{-1}\right)$ allows us to estimate the ratio between the density of the medium and the density of the jet. Using the formulation of Raga et al. (1997), the ratio of ambient medium density, $\rho_{a}$, to jet density, $\rho_{j}$, is

$$
\frac{\rho_{a}}{\rho_{j}}=\left(\frac{v_{j}}{v_{\mathrm{ws}}}-1\right)^{2},
$$

where $v_{j}$ and $v_{\mathrm{ws}}$ are the jet and working surface velocities, respectively. For a jet velocity of $490 \mathrm{~km} \mathrm{~s}^{-1}$ and an upper limit of $160 \mathrm{~km} \mathrm{~s}^{-1}$ for the velocity of the working surface, we obtain $\rho_{a} / \rho_{j} \geqslant 4$.

The precession rate of the jet is $\beta=\alpha v$. Hence with $\alpha=2.3 \pm 0.4 \mathrm{deg} \operatorname{arcsec}^{-1}$, and $v=490 \pm 80 \mathrm{~km} \mathrm{~s}^{-1}$, $\beta=0.080 \pm 0.02 \mathrm{deg} \mathrm{yr}^{-1}$. Note that the measurements of $\alpha$ and $v$ are both $6 \sigma$ results, but the measurement of $\beta$ is a $4 \sigma$ result. The precession period of the jet is $T=2 \pi \theta_{m} /(\alpha v)$, 
which for a precession opening angle of $30^{\circ}$ would be about 5500 years. Since the range in P.A. among the components is about $25^{\circ}$ (see Figure 7), the jet travel time to outer components is about 300 years.

We might expect that the sources excited by the passing jet would persist after the jet moves on. However, there is no evidence (see Figure 8) of structure in the transverse direction in any of the sources. This absence of "trails" suggests that the decay times for emission must be less than $\sim 100$ years, which would produce an extension of $1.5^{\prime \prime}$ at the radius of sources $\mathrm{N}-1$ and $\mathrm{S}-1$. The recombination time for an $\mathrm{H}$ II region with an electron density $n_{e}$ is estimated as

$$
\left(\frac{t}{\mathrm{yr}}\right)=1.2 \times 10^{5}\left(\frac{n_{e}}{\mathrm{~cm}^{-3}}\right)^{-1} .
$$

The spectral index of N-1 indicates it is probably a thermal optically thin H II region (Rodríguez et al. 2005). Using the values for the flux density and size in Table 1 we estimate an emission measure of $1.8 \times 10^{6} \mathrm{~cm}^{-6} \mathrm{pc}^{-1}$ and the electron density as $n_{e}=1.1 \times 10^{4} \mathrm{~cm}^{-3}$. Using this value for the electron density in Equation (10) we find a recombination time for the $\mathrm{N}-1$ component to be about 11 years. This time is an order of magnitude smaller than that required for the jet to leave an observable transverse trail.

For the S-1 source the emission may have a non-thermal component (Rodríguez et al 2005). The density of relativistic electrons, $n_{\mathrm{er}}$, and magnetic field, $B$, can be estimated using Equations (2) and (3) from Garay et al. (1996):

$$
\begin{aligned}
& \left(\frac{B}{\mathrm{mG}}\right)=0.50\left(\frac{S_{v}}{\mathrm{mJy}}\right)^{2 / 7}\left(\frac{\theta_{s}}{\operatorname{arcsec}}\right)^{-6 / 7}\left(\frac{v}{10 \mathrm{GHz}}\right)^{1 / 7} \\
& \times\left(\frac{D}{\mathrm{kpc}}\right)^{-2 / 7}\left(\log \frac{E_{\max }}{E_{\min }}\right)^{2 / 7} \\
& \left(\frac{n_{\mathrm{er}} E_{\min }}{10^{-9} \mathrm{ergs} \mathrm{cm}^{-3}}\right)=3.58\left(\frac{S_{v}}{\mathrm{mJy}}\right)\left(\frac{\theta_{s}}{\operatorname{arcsec}}\right)^{-3}\left(\frac{v}{10 \mathrm{GHz}}\right)^{1 / 2} \\
& \times\left(\frac{D}{\mathrm{kpc}}\right)^{-1}\left(\frac{B}{\mathrm{mG}}\right)^{2 / 7} \text {, }
\end{aligned}
$$

where $E_{\max }$ and $E_{\min }$ are the maximum and minimum energies of the relativistic electrons that we assume are $10^{11}$ and $10^{6} \mathrm{eV}$, respectively. For the distance $D=2.9 \mathrm{kpc}$ and $\theta_{s}=$ 0.76 arcsec, we get $B=1.4 \mathrm{mG}$ and a density of relativistic electrons of $n_{\mathrm{er}}=4.6 \times 10^{-3} \mathrm{~cm}^{-3}$. The decay time is given by Krolik (1999) as $t_{d}=1.3 \times 10^{12} v^{-1 / 2} B^{-3 / 2}$ sec with $v$ in $\mathrm{Hz}$ and $B$ in $\mathrm{G}$, or $t_{d}(\mathrm{yr})=0.5 B^{-3 / 2}$ for our frequency. Using the value derived above for $B$ we get $t_{d}=9 \times 10^{4} \mathrm{yr}$, which is much too long. A magnetic field of $30 \mathrm{mG}$ would be required to achieve a synchrotron decay time of 100 years.

Franco-Hernández et al. (2008, in preparation) have discovered two linear structures traced by water vapor masers on the mas scale. Their $g 2$ group of maser has a P.A. of about $-30^{\circ}$, which is $13^{\circ}$ away from the current P.A. of the proposed precessing source $\left(-43^{\circ}\right)$. Another group of masers $(g l)$ has a P.A. of about $50^{\circ}$. If these masers were in a disk, their pole P.A. would be $-40^{\circ}$, close to the current precession angle. Hence these masers may be associated with the precessing excitation source.

The larger-scale CO lobes are an offset from the jet by about $15^{\prime \prime}$ along a P.A. of about $-6^{\circ}$. The line-of-sight velocity with
Table 3

Jet and Molecular Outflow Parameters of Three Massive Young Stars

\begin{tabular}{lcccc}
\hline \hline Source & $\begin{array}{c}\text { Distance } \\
(\mathrm{kpc})\end{array}$ & $\begin{array}{c}S_{v}(3.6 \mathrm{~cm}) \\
(\mathrm{mJy})\end{array}$ & $\begin{array}{c}\dot{P} \\
\left(M_{\odot} \mathrm{yr}^{-1} \mathrm{~km} \mathrm{~s}^{-1}\right)\end{array}$ & References \\
\hline IRAS 16547-4247 & 2.9 & 8.7 & $4.0 \times 10^{-1}$ & $1,2,3,4$ \\
HH 80-81 & 1.7 & 3.0 & $5.3 \times 10^{-2}$ & 5,6 \\
Cep A HW2 & 0.725 & 6.9 & $5.4 \times 10^{-3}$ & 7,8 \\
\hline
\end{tabular}

References. (1) Garay et al. (2007); (2) Garay et al. (2003); (3) Rodríguez et al. (2005); (4) this paper; (5) Martí et al. (1993); (6) Yamashita et al. (1989); (7) Curiel et al. (2006); (8) Narayanan \& Walker (1996).

respect to the ambient cloud is about $\pm 30 \mathrm{~km} \mathrm{~s}^{-1}$. These lobes could also be associated with the precessing source. The expected P.A. for emission at $15^{\prime \prime}$ from Equation (7) would be about $-8^{\circ}$, close to the observed value. If the molecular flow picks up the full jet velocity of $490 \mathrm{~km} \mathrm{~s}^{-1}$, then the inclination would be about $90-$ arctan $(30 / 490)$, or about $86^{\circ}$, very close to the value inferred from the analysis of the velocity structure of the CO lobes (Garay et al 2007). More realistically, the molecular flow velocity will be smaller, implying a smaller inclination angle.

The precession model nicely accounts for the fact that the line from N-1 to S-1 does not intercept the known jet. However, if the angle of the driving precessing source is currently $-43^{\circ}$, it is significantly discrepant with the P.A. of $-16^{\circ}$ of the known radio jet. A possible explanation for this discrepancy might be that the central source is really a binary stellar system. Source I would be a non-precessing source associated with the known jet, which is related to the large-scale CO outflow with about the same P.A. Source II would be a precessing source which drives the continuum thermal sources and may be associated with the water masers and also the large-scale CO outflow.

\section{PUTTING THE IRAS 16547-4247 JET IN CONTEXT}

How does the IRAS 16547-4247 jet compare with other jets found in regions of star formation? Anglada et al. (1992) have compared the centimeter radio luminosity (taken to be proportional to the flux density times the distance squared, $S_{v} d^{2}$ ) of thermal jets associated with low-mass young stars with the momentum rate $(\dot{P})$ in the associated molecular outflow. For 16 sources studied, they conclude that these low-mass objects can be fitted with a power law of the form $\dot{P}=10^{-2.6}\left(S_{v} d^{2}\right)^{1.1}$, where $\dot{P}$ is in $M_{\odot} \mathrm{yr}^{-1} \mathrm{~km} \mathrm{~s}^{-1}, S_{v}$ is in mJy and $d$ is in kpc. In Figure 9, we plot the data used by Anglada et al. (1992). They interpret the fit to be in agreement with a simple model in which the observed ionization is produced by shocks (Curiel et al. 1987, 1989), where about $10 \%$ of the energy in the jet is thermalized. We have plotted in the same figure, the three beststudied cases of thermal jets associated with massive young stars: IRAS 16547-4247, HH 80-81, and Cep A HW2, using the data listed in Table 3. Remarkably, these three data points fall reasonably well on the Anglada et al. correlation. We believe that this agreement suggests that the jets associated with massive young stars may be a scaled-up version of the phenomenon seen in low-mass young stars, although a firmer conclusion requires the study of a larger sample of objects than that available now. Note that the intrinsic radio luminosity of the IRAS 16547-4247 jet is about $2 \times 10^{3}$ larger than the typical radio luminosity of jets associated with low-mass stars, and that it is the most luminous case known. 


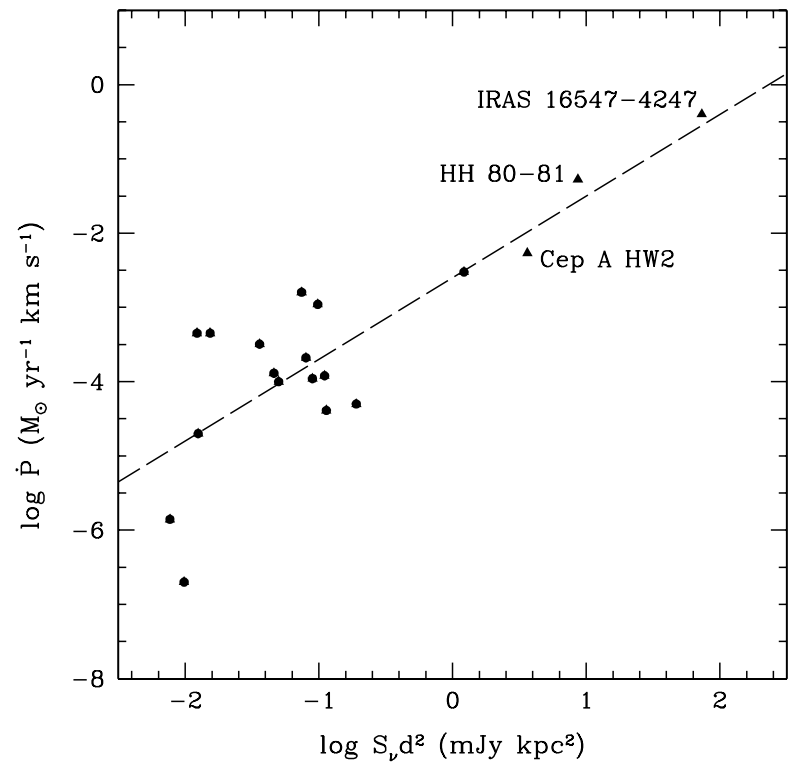

Figure 9. Momentum rate in the molecular outflow, $\dot{P}$, versus the radio fluxdensity times distance squared, $S_{\nu} d^{2}$. The solid dots are the 16 low-mass young stars from the study of Anglada et al. (1992). We have included three high-mass young stars (solid triangles, see also labels in figure) from this paper as well as from the references given in Table 3. The dashed line is the fit of Anglada et al. (1992) to the low-mass young stars. The high-mass young stars fall reasonably well on the correlation, suggesting a common nature for thermal jets associated with low- and high-mass young stars.

\section{CONCLUSIONS}

Our main conclusions are as follows.

1. We present new, sensitive $3.6 \mathrm{~cm}$ wavelength VLA observations of the multiple radio source associated with the luminous infrared source IRAS 16547-4247, the most massive example known of a thermal jet found in association with a forming star. The main purposes of these new observations were to search for variability and proper motions as well as to detect additional faint sources in the region. We detected four new components $(\mathrm{N}-4, \mathrm{~N}-5, \mathrm{D}$, and $\mathrm{E})$ in the region.

2. We do not detect proper motions along the axis of the flow in the outer lobes of this source at a $4 \sigma$ upper limit of $\sim 160 \mathrm{~km}$ $\mathrm{s}^{-1}$, suggesting that if these lobes are the working surfaces of the jets and the jet velocity is $\sim 490 \mathrm{~km} \mathrm{~s}^{-1}$, the ambient medium is at least four times as dense as the jet.

3. The brightest components of the lobes, sources N-1 and $\mathrm{S}-1$, show evidence of clockwise precession, at a rate of $0.08 \mathrm{yr}^{-1}$ in the plane of the sky.

4. A precessing model can account for the antisymmetric distribution of most of the sources in the field, as well as for the evidence of precession in sources N-1 and S- 1 .

5. The thermal jet at the core of the region shows significant variations in flux density and morphology but our angular resolution is insufficient to reach a clear conclusion on what produces these changes.

6. The correlation found by Anglada et al. (1992) for outflows and jets in low-mass stars extends to the handful of massive forming stars known. This result suggests that the jets associated with massive young stars are a scaled-up version of the phenomenon seen in low-mass young stars, although a firmer conclusion requires the study of a larger sample of jets than that currently available.

We thank an anonymous referee for valuable comments. L.F.R. acknowledges support from grant CB0702172_3 of COECyT, Michoacán, México. D.M. and G.G. acknowledge support from the Chilean Centro de Astrofísica FONDAP 15010003

\section{REFERENCES}

Anglada, G. 1996, in ASP Conf. Ser. 93, Radio Emission from the Stars and the Sun, ed. A. R. Taylor, \& J. M. Paredes (San Francisco, CA: ASP), 3

Anglada, G., Rodríguez, L. F., Cantó, J., Estalella, R., \& Torrelles, J. M. 1992, ApJ, 395, 494

Arce, H. G., Shepherd, D., Gueth, F., Lee, C.-F., Bachiller, R., Rosen, A., \& Beuther, H. 2007, in Protostars and Planets V, ed. B. Reipurth, D. Jewitt, \& K. Keil (Tucson, AZ: Univ. Arizona Press), 245

Bally, J., \& Zinnecker, H. 2005, AJ, 129, 2281

Bonnell, I. A., Bate, M. R., \& Zinnecker, H. 1998, MNRAS, 298, 93

Brand, J., \& Blitz, L. 1993, A\&A, 275, 67

Brooks, K. J., Garay, G., Mardones, D., \& Bronfman, L. 2003, ApJ, 594, L131

Brooks, K. J., Garay, G., Voronkov, M., \& Rodríguez, L. F. 2007, ApJ, 669, 459

Caswell, J. L. 1998, MNRAS, 297, 215

Cesaroni, R., Galli, D., Lodato, G., Walmsley, C. M., \& Zhang, Q. 2007, in Protostars and Planets V, ed. B. Reipurth, D. Jewitt, \& K. Keil (Tucson, AZ: Univ. Arizona Press), 197

Chakrabarti, S. K. 1988, MNRAS, 235, 33

Curiel, S., Cantó, J., \& Rodríguez, L. F. 1987, RevMexA\&A, 14, 595

Curiel, S., Rodríguez, L. F., Bohigas, J., Roth, M., Cantó, J., \& Torrelles, J. M. 1989, ApL\&C, 27, 299

Curiel, S., et al. 2006, ApJ, 638, 878

Eislöffel, J., Mundt, R., Ray, T. P., \& Rodríguez, L. F. 2000, in Protostars and Planets IV, ed. V. Mannings, A. P. Boss, \& S. S. Russell (Tucson, AZ: Univ. Arizona Press), 815

Forster, J. R., \& Caswell, J. L. 1989, A\&A, 213, 339

Galván-Madrid, R., Avila, R., \& Rodríguez, L. F. 2004, RevMexA\&A, 40, 31

Garay, G., Brooks, K. J., Mardones, D., \& Norris, R. P. 2003, ApJ, 587, 739

Garay, G., \& Lizano, S. 1999, P.A.SP, 111, 1049

Garay, G., Mardones, D., Bronfman, L., Brooks, K. J., Rodríguez, L. F., Güsten, R., Nyman, L-Ä, Franco-Hernández, R., \& Moran, J. M. 2007, A\&A, 463, 217

Garay, G., Ramirez, S., Rodríguez, L. F., Curiel, S., \& Torrelles, J. M. 1996, ApJ, 459, 193

Krolik, J. H. 1999, Active Galactic Nuclei: From the Central Black Hole to the Galactic Environment (Princeton, NJ: Princeton Univ. Press)

Martí, J., Rodríguez, L. F., \& Reipurth, B. 1993, ApJ, 416, 208

Martí, J., Rodríguez, L. F., \& Reipurth, B. 1995, ApJ, 449, 184

Martí, J., Rodríguez, L. F., \& Reipurth, B. 1998, ApJ, 502, 337

Narayanan, G., \& Walker, C. F. 1996, ApJ, 466, 844

Raga, A. C., Rodríguez, L. F., \& Cantó, J. 1997, RMxF, 43, 825

Ray, T., Dougados, C., Bacciotti, F., Eislöffel, J., \& Chrysostomou, A. 2007, in Protostars and Planets V, ed. B. Reipurth, D. Jewitt, \& K. Keil (Tucson, AZ: Univ. Arizona Press), 231

Rodríguez, L. F. 1997, in Proc. of IAU Symp. 182, Herbig-Haro Flows and the Birth of low-mass Stars, ed. B. Reipurth, \& C. Bertout (Dordrecht: Kluwer), 83

Rodríguez, L. F., Delgado Arellano, V. G., Gómez, Y., Reipurth, B., Torrelles, J. M., Noriega-Crespo, A., Raga, A. C., \& Cantó, J. 2000, AJ, 119, 882

Rodríguez, L. F., Garay, G., Brooks, K. J., \& Mardones, D. 2005, ApJ, 626, 953 Rodríguez, L. F., Torrelles, J. M., Anglada, G., \& Martí, J. 2001, RevMexA\&A, 37,95

Shu, F. H., Adams, F. C., \& Lizano, S. 1987, ARA\&A, 25, 23

Stahler, S. W., Palla, F., \& Ho, P. T. P. 2000, in Protostars and Planets IV, ed. V. Mannings, A. P. Boss, \& S. S. Russell (Tucson, AZ: Univ. Arizona Press), 327

Stark, A. A., \& Brand, J. 1989, ApJ, 339, 763

Voronkov, M. A., Brooks, K. J., Sobolev, A. M., Ellingsen, S. P., Ostrovskii, A. B., \& Caswell, J. L. 2006, MNRAS, 373, 411

Windhorst, R. A., Fomalont, E. B., Partridge, R. B., \& Lowenthal, J. D. 1993, ApJ, 405, 498

Yamashita, T., Suzuki, H., Kaifu, N., Tamura, M., Mountain, C. M., \& Moore, T. J. T. 1989, ApJ, 347, 894 